\title{
Steffensen-Type Method of Super Third-Order Convergence for Solving Nonlinear Equations
}

\author{
Zhongli Liu*, Hong Zhang \\ College of Biochemical Engineering, Beijing Union University, Beijing, China \\ Email: "liuzhongli2@163.com
}

Received 5 March 2014; revised 5 April 2014; accepted 12 April 2014

Copyright (C) 2014 by authors and Scientific Research Publishing Inc.

This work is licensed under the Creative Commons Attribution International License (CC BY).

http://creativecommons.org/licenses/by/4.0/

(c) (i) Open Access

\begin{abstract}
In this paper, a one-step Steffensen-type method with super-cubic convergence for solving nonlinear equations is suggested. The convergence order 3.383 is proved theoretically and demonstrated numerically. This super-cubic convergence is obtained by self-accelerating second-order Steffensen's method twice with memory, but without any new function evaluations. The proposed method is very efficient and convenient, since it is still a derivative-free two-point method. Its theoretical results and high computational efficiency is confirmed by Numerical examples.
\end{abstract}

\section{Keywords}

Newton's Method, Steffensen's Method, Derivative Free, Super-Cubic Convergence, Nonlinear Equation

\section{Introduction}

Finding the root of a nonlinear equation

$$
f(x)=0
$$

is a classical problem. It is well-known in scientific computation that Newton's method (NM, see [1]):

$$
x_{n+1}=x_{n}-\frac{f\left(x_{n}\right)}{f^{\prime}\left(x_{n}\right)}, \quad n=0,1,2, \cdots,
$$

is widely used for root-finding, where $x_{0}$ is an initial guess of the root. However, when the derivative $f^{\prime}$ is "Corresponding author.

How to cite this paper: Liu, Z.L. and Zhang, H. (2014) Steffensen-Type Method of Super Third-Order Convergence for Solving Nonlinear Equations. Journal of Applied Mathematics and Physics, 2, 581-586. 
unavailable or is expensive to be obtained, the derivative-free method is necessary. If the derivative $f^{\prime}\left(x_{n}\right)$ is replaced by the divided difference in (2), Steffensen's method (SM, see [1]) is obtained as follows:

$$
x_{n+1}=x_{n}-\frac{f\left(x_{n}\right)}{f\left[x, x_{n}+f\left(x_{n}\right)\right]}, \quad n=0,1,2, \cdots,
$$

$\mathrm{NM} / \mathrm{SM}$ converges quadratically and requires two function evaluations per iteration. The efficiency index of them is $\sqrt{2}=1.414$.

Besides H.T. Kung and J.F. Traub conjectured that an iterative method based on $m$ evaluations per iteration without memory would arrive at the optimal convergence of order $2^{m-1}$ (see [2]), Traub proposed a self-accelerating two-point method of order 2.414 with memory (see [3]):

$$
\left\{\begin{array}{l}
x_{n+1}=x_{n}-\frac{f\left(x_{n}\right)}{f\left[x_{n}+\beta_{n} f\left(x_{n}\right)\right]}, \\
\beta_{n}=-\frac{1}{f\left[x_{n}, z_{n-1}\right]}
\end{array}\right.
$$

where $z_{n-1}=x_{n-1}+\beta_{n-1} f\left(x_{n-1}\right)$, and $\beta_{0}=-\operatorname{sign}\left(f^{\prime}\left(x_{0}\right)\right)$ or $-1 / f\left[x_{0}, x_{0}+f\left(x_{0}\right)\right]$, etc.

A lot of self-accelerating Steffensen-type methods were derived in the literature (see [1]-[7]). Steffensen-type methods and their applications in the solution of nonlinear systems and nonlinear differential equations were discussed in [1] [4] [5] [8]. Recently, by a new self-accelerating technique based on the second-order Newtonian interpolatory polynomial $N_{2}(x)=f\left(x_{n}\right)+f\left[x_{n}, z_{n-1}\right]\left(x-x_{n}\right)+f\left[x_{n}, z_{n-1}, x_{n-1}\right]\left(x-x_{n}\right)\left(x-z_{n-1}\right)$, J. Džunića and M.S. Petkovića proposed a cubically convergent Steffensen-like method (see [7]):

$$
\left\{\begin{array}{l}
x_{n+1}=x_{n}-\frac{f\left(x_{n}\right)}{f\left[x_{n}, x_{n}+\beta_{n} f\left(x_{n}\right)\right]}, \\
\beta_{n}=-\frac{1}{f\left[x_{n}, z_{n-1}\right]+f\left[x_{n}, x_{n-1}\right]-f\left[x_{n-1}, z_{n-1}\right]},
\end{array}\right.
$$

In this study, a one-step Steffensen-type method is proposed by doubly-self-accelerating in Section 2, its super-cubic convergence is proved in Section 3, and numerical examples are demonstrated in Section 4.

\section{The Method of Steffensen-Type}

By the first-order Newtonian interpolatory polynomial $N_{1}(x)=f\left(x_{n}\right)+f\left[x_{n}, z_{n}\right]\left(x-x_{n}\right)$ and

$$
z_{n}=x_{n}+\beta_{n} f\left(x_{n}\right) \text {, }
$$

we have $f(x)=N_{1}(x)+R_{1}(x)$,

where

$$
R_{1}(x)=f(x)-N_{1}(x)=f\left[x_{n}, z_{n}, x\right]\left(x-x_{n}\right)\left(x-z_{n}\right) .
$$

So, with some $\mu_{n} \approx f\left[x_{n}, z_{n}, x\right]$,

$$
\tilde{N}_{2}(x)=f\left(x_{n}\right)+f\left[x_{n}, z_{n}\right]\left(x-x_{n}\right)+\mu_{n}\left(x-x_{n}\right)\left(x-z_{n}\right)
$$

should be better than $N_{1}(x)$ to approximate $f(x)$.

Therefore, we suggest $x_{n+1}=x_{n}-\frac{\tilde{N}_{2}\left(x_{n}\right)}{N_{2}^{\prime}\left(x_{n}\right)}$, i.e., a two-parameter Steffensen's method:

$$
x_{n+1}=x_{n}-\frac{f\left(x_{n}\right)}{f\left[x_{n}, z_{n}\right]+\mu\left(x_{n}-z_{n}\right)}, \quad n=0,1,2, \cdots,
$$


where $z_{n}=x_{n}+\beta_{n} f\left(x_{n}\right),\left\{\beta_{n}\right\}$ and $\left\{\mu_{n}\right\}$ are bounded constant sequences. The error equation of (7) is $e_{n+1}=\left[\left(1+\beta_{n} f^{\prime}(a)\right) \frac{f^{\prime \prime}(a)}{2 f^{\prime}(a)}-\mu_{n} \beta_{n}\right] e_{n}^{2}+O\left(e_{n}^{3}\right)$. By defining $\mu_{0}=0$ and $\mu_{n}=\frac{1+\beta_{n} f\left[x_{n}, z_{n}\right]}{\beta_{n} f\left[x_{n}, z_{n}\right]} f\left[z_{n-1}, x_{n}, z_{n}\right](n>0)$ recursively as the iteration proceeds without any new evaluation to vanish the asymptotic convergence constant, we establish a self-accelerating Steffensen's method with super quadratic convergence as follows:

$$
x_{n+1}=x_{n}-\frac{f\left(x_{n}\right)}{f\left[x_{n}, z_{n}\right]-\left(1+\frac{1}{\beta_{n} f\left[x_{n}, z_{n}\right]}\right)\left(f\left[z_{n-1}, z_{n}\right]-f\left[x_{n}, z_{n-1}\right]\right)}, \quad n=0,1,2, \cdots,
$$

Furthermore, we propose a one-step Steffensen-type method with super cubic convergence by doubly-selfaccelerating as follows:

$$
\left\{\begin{array}{l}
x_{n+1}=x_{n}-\frac{f\left(x_{n}\right)}{f\left[x_{n}, z_{n}\right]-\left(1+1 /\left(\beta_{n} f\left[x_{n}, z_{n}\right]\right)\right)\left(f\left[z_{n-1}, z_{n}\right]-f\left[x_{n}, z_{n-1}\right]\right)} \\
\beta_{n}=\frac{1}{f\left[x_{n-1}, z_{n-1}\right]-f\left[x_{n}, z_{n-1}\right]-f\left[x_{n-1}, x_{n}\right]}
\end{array}\right.
$$

\section{Its Super Third-Order Convergence}

Lemma $3.1 \beta_{n} \sim-\frac{1}{f^{\prime}(a)} f\left(1+c_{3} e_{n-1} e_{n-1}^{z}\right)$, where $c_{k}=\frac{f^{(k)}(a)}{k ! f^{\prime}(a)}, \quad e_{n}=x_{n}-a$ and $e_{n}^{z}=z_{n}-a$.

Proof. By Taylor formula, we have

$$
\begin{aligned}
& f\left[x_{n}, z_{n-1}\right]+f\left[x_{n-1}, x_{n}\right]-f\left[x_{n-1}, z_{n-1}\right] \\
= & \frac{f\left(x_{n}\right)-f\left(x_{n-1}\right)}{x_{n}-x_{n-1}}+\frac{f\left(x_{n}\right)-f\left(z_{n-1}\right)}{x_{n}-z_{n-1}}-\frac{f\left(z_{n-1}\right)-f\left(x_{n-1}\right)}{z_{n-1}-x_{n-1}} \\
= & \frac{f\left(x_{n}\right)-f\left(x_{n-1}\right)}{e_{n}-e_{n-1}}+\frac{f\left(x_{n}\right)-f\left(z_{n-1}\right)}{e_{n}-e_{n-1}^{z}}-\frac{f\left(z_{n-1}\right)-f\left(x_{n-1}\right)}{e_{n-1}^{z}-e_{n-1}} \\
= & f^{\prime}(a)\left[\frac{e_{n}-e_{n-1}+c_{2}\left(e_{n}^{2}-e_{n-1}^{2}\right)+c_{3}\left(e_{n}^{3}-e_{n-1}^{3}\right)+\cdots}{e_{n}-e_{n-1}}\right. \\
& +\frac{e_{n}-e_{n-1}^{z}+c_{2}\left(e_{n}^{2}-\left(e_{n-1}^{z}\right)^{2}\right)+c_{3}\left(e_{n}^{3}-\left(e_{n-1}^{z}\right)^{3}\right)+\cdots}{e_{n}-e_{n-1}^{z}} \\
& \left.-\frac{\left.e_{n-1}^{z}-e_{n-1}+c_{2}\left(\left(e_{n-1}^{z}\right)^{2}-e_{n-1}^{2}\right)+c_{3}\left(\left(e_{n-1}^{z}\right)^{3}-e_{n-1}^{3}\right)+\cdots\right]}{e_{n-1}^{z}-e_{n-1}}\right] \\
\sim & f^{\prime}(a)\left(1-c_{3} e_{n-1} e_{n-1}^{z}\right) .
\end{aligned}
$$

So,

$$
\beta_{n}=\frac{1}{f\left[x_{n-1}, z_{n-1}\right]-f\left[x_{n}, z_{n-1}\right]-f\left[x_{n}, x_{n-1}\right]} \sim-\frac{1}{f^{\prime}(a)} f\left(1+c_{3} e_{n-1} e_{n-1}^{z}\right) .
$$

Then, the proof can be completed. 
Theorem 3.2 Let $f: D \rightarrow R$ be a sufficiently differentiable function with simple root $a \in D, D \subset R$ be an open set, $x_{0}$ be close enough to $a$, then (9) achieve the convergence of order 3.383 .

Proof. If $z_{n}$ converges to $a$ with order $p>1$ as:

$$
e_{n}^{z}=C_{n} e_{n}^{p}+o\left(e_{n}^{p}\right)
$$

and if $x_{n}$ converges to $a$ with order $r>2$ as:

$$
e_{n+1}=D_{n} e_{n}^{r}+o\left(e_{n}^{r}\right),
$$

Then

$$
\begin{gathered}
e_{n}^{z}=C_{n}\left(D_{n-1} e_{n-1}^{r}\right)^{p}+o\left(e_{n-1}^{r p}\right)=C_{n} D_{n-1}^{p} e_{n-1}^{r p}+o\left(e_{n-1}^{r p}\right), \\
e_{n+1}=D_{n}\left(D_{n-1} e_{n-1}^{r}\right)^{r}+o\left(e_{n-1}^{r^{2}}\right)=D_{n} D_{n-1}^{r} e_{n-1}^{r^{2}}+o\left(e_{n-1}^{r^{2}}\right) .
\end{gathered}
$$

By Taylor formula and Lemma 3.1, we also have

$$
\begin{gathered}
e_{n}^{z}=\left(1+\beta_{n} f\left[x_{n}, a\right]\right) e_{n}=-c_{3} e_{n-1} C_{n-1} e_{n-1}^{p} D_{n-1} e_{n-1}^{r}+o\left(e_{n-1}^{r+p+1}\right) \\
=--c_{3} C_{n-1} D_{n-1} e_{n-1}^{r+p+1}+o\left(e_{n-1}^{r+p+1}\right) . \\
e_{n+1}=e_{n}-\frac{f\left[x_{n}, a\right] e_{n}}{f\left[x_{n}, z_{n}\right]-\left(1+1 /\left(\beta_{n} f\left[x_{n}, z_{n}\right]\right)\right)\left(f\left[z_{n-1}, z_{n}\right]-f\left[z_{n-1}, x_{n}\right]\right)} \\
=e_{n} \frac{f\left[x_{n}, z_{n}\right]+\left(1+1 /\left(\beta_{n} f\left[x_{n}, z_{n}\right]\right)\right)\left(f\left[z_{n-1}, x_{n}\right]-f\left[z_{n-1}, z_{n}\right]\right)-f\left[x_{n}, a\right]}{f\left[x_{n}, z_{n}\right]-\left(1+1 /\left(\beta_{n} f\left[x_{n}, z_{n}\right]\right)\right)\left(f\left[z_{n-1}, z_{n}\right]-f\left[z_{n-1}, x_{n}\right]\right)} \\
=e_{n} \frac{f\left[x_{n}, z_{n}, a\right] e_{n}^{z}+\left(1+1 /\left(\beta_{n} f\left[x_{n}, z_{n}\right]\right)\right) f\left[z_{n-1}, x_{n}, z_{n}\right]\left(-\beta_{n} f\left[x_{n}, a\right]\right)}{f\left[x_{n}, z_{n}\right]-\left(1+1 /\left(\beta_{n} f\left[x_{n}, z_{n}\right]\right)\right)\left(f\left[z_{n-1}, z_{n}\right]-f\left[z_{n-1}, x_{n}\right]\right)} \\
=e_{n} \frac{f\left[x_{n}, z_{n}, a\right]\left(1+\beta_{n} f\left[x_{n}, a\right]\right) e_{n}-\left(1+\beta_{n} f\left[x_{n}, z_{n}\right]\right)-\frac{f\left[x_{n}, a\right]}{f\left[x_{n}, z_{n}\right]} f\left[z_{n-1}, x_{n}, z_{n}\right] e_{n}}{f\left[x_{n}, z_{n}\right]-\left(1+1 /\left(\beta_{n} f\left[x_{n}, z_{n}\right]\right)\right)\left(f\left[z_{n-1}, z_{n}\right]-f\left[z_{n-1}, x_{n}\right]\right)} \\
=e_{n}^{2}\left(1+\beta_{n} f\left[x_{n}, a\right]\right) \frac{f\left[x_{n}, z_{n}, a\right] f\left[x_{n}, z_{n}\right]-f\left[x_{n}, a\right] f\left[z_{n-1}, x_{n}, z_{n}\right]}{f^{2}\left[x_{n}, z_{n}\right]-\left(1+1 /\left(\beta_{n} f\left[x_{n}, z_{n}\right]\right)\right) f\left[x_{n}, z_{n}\right]\left(f\left[z_{n-1}, z_{n}\right]-f\left[z_{n-1}, x_{n}\right]\right)} \\
=e_{n}^{2}\left(1+\beta_{n} f\left[x_{n}, a\right]\right) \frac{f^{2}\left[x_{n}, z_{n}, a\right] e_{n}^{z}-f\left[x_{n}, a\right] f\left[z_{n-1}, x_{n}, z_{n}\right] e_{n-1}^{z}}{f^{2}\left[x_{n}, z_{n}\right]-\left(1+1 /\left(\beta_{n} f\left[x_{n}, z_{n}\right]\right)\right) f\left[x_{n}, z_{n}\right]\left(f\left[z_{n-1}, z_{n}\right]-f\left[z_{n-1}, x_{n}\right]\right)} \\
=e_{n}^{2}\left(-c_{3} e_{n-1} e_{n-1}^{z}+\cdots\right) \frac{-f^{\prime}(a) \frac{f^{\prime \prime \prime}(a)}{3 !} e_{n-1}^{z}+\cdots}{f^{\prime 2}(a)+\cdots}
\end{gathered}
$$

So, comparing the exponents of $e_{n-1}$ in expressions of $e_{n}^{z}$ and $e_{n+1}$ for (9), we obtain the same system of two equations:

$$
\left\{\begin{array}{l}
r p=r+p+1, \\
r^{2}=2 r+2 p+1 .
\end{array}\right.
$$

From its non-trivial solution $r \approx 3.383$ and $p \approx 1.839$, we prove that the convergence of (9) is of order 3.383 .

As the efficiency index is $p^{1 / w}$, without any additional function evaluations, the efficiency indices of (4), (5) and (9) are $\sqrt{1+\sqrt{2}}=1.554, \sqrt{3}=1.732$ and $\sqrt{3.383}=1.839$, respectively. 


\section{Numerical Examples}

Related one-step methods only using two function evaluations per iteration are showed in the following numerical examples. The proposed method is a derivative-free two-point method with high computational efficiency.

Example 1. The numerical results of NM, SM, (4), (5) and (9) in Table 1 agree with the theoretical analysis. The computational order of convergence is defined by

$$
\mathrm{COC}=\frac{\log \left(\left|e_{n}\right| /\left|e_{n-1}\right|\right)}{\log \left(\left|e_{n-1}\right| /\left|e_{n-2}\right|\right)} .
$$

Example 2. The numerical results of NM, SM, (4), (5) and (9) are in Table 2 for the following nonlinear functions:

$$
\begin{gathered}
f_{1}(x)=0.5\left(\mathrm{e}^{x-2}-1\right), a=2, x_{0}=2.5, \\
f_{2}(x)=\mathrm{e}^{x^{2}}+\sin x-1, a=0, x_{0}=0.25, \\
f_{3}(x)=\mathrm{e}^{-x^{2}+x+2}-1, \quad a=-1, x_{0}=-0.85, \\
f_{4}(x)=\mathrm{e}^{-x}-\arctan x-1, \quad a=0, x_{0}=-0.2 .
\end{gathered}
$$

\begin{tabular}{|c|c|c|c|c|c|}
\hline Methods & NM & SM & (4) & (5) & (9) \\
\hline$f_{1}:\left|e_{6}\right|$ & $0.19785 e^{-40}$ & $0.88156 e^{-29}$ & $0.50439 \mathrm{e}-84$ & $0.19314 e^{-}-313$ & $0.75162 e^{-578}$ \\
\hline COC & 2.0000 & 2.0000 & 2.4141 & 3.0000 & 3.3831 \\
\hline$f_{2}:\left|e_{6}\right|$ & $0.32328 e^{-44}$ & $0.42920 e^{-26}$ & $0.19843 e-85$ & $0.57587 e^{-}-282$ & $0.13494 e^{-706}$ \\
\hline $\mathrm{COC}$ & 2.0000 & 2.0000 & 2.4141 & 3.0000 & 3.3825 \\
\hline$f_{3}:\left|e_{6}\right|$ & $0.18813 e^{-51}$ & $0.15758 e^{-18}$ & $0.12013 e-86$ & $0.34524 \mathrm{e}^{-}-286$ & $0.27679 \mathrm{e}-677$ \\
\hline COC & 2.0000 & 2.0000 & 2.4140 & 3.0000 & 3.3796 \\
\hline$f_{4}:\left|e_{6}\right|$ & $0.35988 e^{-79}$ & $0.96290 e^{-}-84$ & $0.16834 \mathrm{e}^{-}-248$ & $0.21536 e^{-597}$ & $0.25291 e^{-1154}$ \\
\hline COC & 2.0000 & 2.0000 & 2.4161 & 3.0000 & 3.3831 \\
\hline
\end{tabular}

Table 1. $f(x)=x^{2}-\mathrm{e}^{-x}-3 x+1, a=0, x_{0}=0.2$.

\begin{tabular}{cccccccc}
\hline Methods & $\mathrm{n}$ & 1 & 2 & 3 & 4 & 5 & 6 \\
\hline $\mathrm{NM}$ & $x_{n}-a \mid$ & $0.53279 \mathrm{e}-2$ & $0.35561 \mathrm{e}-5$ & $0.15808 \mathrm{e}-11$ & $0.31235 \mathrm{e}-24$ & $0.12195 \mathrm{e}-49$ & $0.15890 \mathrm{e}-100$ \\
& $\mathrm{COC}$ & 2.25256 & 2.01691 & $0.15808 \mathrm{e}-11$ & 2.00000 & 2.00000 & 2.00000 \\
$\mathrm{SM}$ & $\left|x_{n}-a\right|$ & $0.28174 \mathrm{e}-1$ & $0.51325 \mathrm{e}-3$ & $0.16476 \mathrm{e}-6$ & $0.16966 \mathrm{e}-13$ & $0.17989 \mathrm{e}-27$ & $0.20226 \mathrm{e}-55$ \\
& COC & 1.21776 & 2.04376 & 2.00830 & 2.00009 & 2.00000 & 2.00000 \\
$(4)$ & $\left|x_{n}-a\right|$ & $0.28174 \mathrm{e}-1$ & $0.15996 \mathrm{e}-4$ & $0.13132 \mathrm{e}-12$ & $0.43283 \mathrm{e}-32$ & $0.38442 \mathrm{e}-79$ & $0.99936-193$ \\
& COC & 1.21776 & 3.81335 & 2.49109 & 2.40945 & 2.41512 & 2.41406 \\
$(5)$ & $\left|x_{n}-a\right|$ & $0.28174 \mathrm{e}-1$ & $0.16560 \mathrm{e}-6$ & $0.11521 \mathrm{e}-21$ & $0.39821 \mathrm{e}-67$ & $0.16444 \mathrm{e}-203$ & $0.11580 \mathrm{e}-612$ \\
& COC & 1.21776 & 6.14536 & 2.89776 & 2.99925 & 3.00000 & 3.00000 \\
$(9)$ & $\mid x_{n}-a$ & $0.28174 \mathrm{e}-1$ & $0.43010 \mathrm{e}-7$ & $0.21604 \mathrm{e}-27$ & $0.23153 \mathrm{e}-94$ & $0.20021 \mathrm{e}-321$ & $0.69689 \mathrm{e}-1090$ \\
& COC & 1.21776 & 6.83322 & 3.49004 & 3.29917 & 3.39052 & 3.38434 \\
\hline
\end{tabular}

Table 2. Numerical results for solving $f_{i}(x), i=1,2,3,4$. 


\section{Conclusion}

By theoretical analysis and numerical experiments, we confirm that the proposed method which is a derivativefree two-point method has high computational efficiency. Its convergence order is 3.383 and its efficiency index is 1.839 . We can see that the suggested method is suitable to solve nonlinear equations and can also be used for solving boundary-value problems of nonlinear ordinary differential equations.

\section{References}

[1] Ortega, J.M. and Rheinboldt, W.G. (1970) Iterative Solution of Nonlinear Equations in Several Variables. Academic Press, New York.

[2] Kung, H.T. and Traub, J.F. (1974) Optimal Order of One-Point and Multipoint Iteration. Journal of the ACM, 21, 634-651. http://dx.doi.org/10.1145/321850.321860

[3] Traub, J.F. (1964) Iterative Methods for the Solution of Equations. Prentice-Hall, Englewood Cliffs.

[4] Zheng, Q., Wang, J., Zhao, P. and Zhang, L. (2009) A Steffensen-Like Method and Its Higher-Order Variants. Applied Mathematics and Computation, 214, 10-16. http://dx.doi.org/10.1016/j.amc.2009.03.053

[5] Zheng, Q., Zhao, P., Zhang, L. and Ma, W. (2010) Variants of Steffensen-Secant Method and Applications. Applied Mathematics and Computation, 216, 3486-3496. http://dx.doi.org/10.1016/j.amc.2010.04.058

[6] Petković, M.S., Ilić, S. and Džunić, J. (2010) Derivative Free Two-Point Methods with and without Memory for Solving Nonlinear Equations. Applied Mathematics and Computation, 217, 1887-1895. http://dx.doi.org/10.1016/j.amc.2010.06.043

[7] Džunić, J. and Petković, M.S. (2012) A Cubically Convergent Steffensen-Like Method for Solving Nonlinear Equations. Applied Mathematics Letters, 25, 1881-1886.

[8] Alarcón, V., Amat, S., Busquier, S. and López, D.J. (2008) A Steffensen’s Type Method in Banach Spaces with Applications on Boundary-Value Problems. Journal of Computational and Applied Mathematics, 216, 243-250. http://dx.doi.org/10.1016/j.cam.2007.05.008 